\title{
Multi-technique, Multivariate Analysis Methods for Enhanced Sample Characterization
}

\author{
K. Artyushkova*, S. Pylypenko*, J. Fenton*, K.Archuleta*, L. Williams** and J. Fulghum* \\ * Chemical and Nuclear Engineering Department, University of New Mexico, Albuquerque, NM \\ 87131 \\ ** Department of Computer Science, University of New Mexico, Albuquerque, NM 87123
}

Recent instrumentation developments have improved spatial resolution and decreased acquisition times for a range of techniques used for materials characterization. Rapid image acquisition has, for example, transformed laboratory XPS imaging from a novelty to an increasingly routine analysis method for surface chemical characterization on the scale of microns [1]. The combination of high spatial and high-energy resolution XPS enhances chemical characterization of heterogeneous samples at the cost of increasing complexity in the data sets acquired. In many cases, there are a variety of approaches possible to not only data analysis, but also data acquisition. Choices made in the data acquisition step, obviously have a significant impact on the data analysis options. A variety of multivariate analysis methods can be used to facilitate analysis of multi-dimensional data sets. The application of multivariate methods in the analysis of XPS datasets encompasses topography correction in images, identification of major and minor chemical components, improvement of spectral and, potentially, spatial resolution and enhanced data visualization. Considerations for data acquisition and processing will be discussed, using examples including surface modified polymers, attached nucleotides, and electronic materials.

Characterization of heterogeneous samples frequently requires multi-technique correlations. The ability to acquire images from the same area on samples using multiple techniques provides opportunities for enhanced sample characterization, including using data from one technique to facilitate or confirm interpretation of data from a second technique. A variety of techniques, including AFM, FTIR and confocal microscopy (CM) have fields-of-view that are comparable to XPS, although spatial resolution and information content differ dramatically.[1,2] We will show a variety of examples of multi-technique correlations including:

- Visualization of 3-D polymer chemistry through correlation of XPS, CM and AFM data (Figure 1)

- Fusion of high spatial resolution AFM images with high energy resolution XPS images for enhanced spatial distribution information

- Quantification of CM image data sets through fusion with XPS quantitative images

- Correlation of AFM images with contact angle data

Correlating the data from multiple techniques, as in the examples listed above, requires matching and marking of the sample analysis areas, image registration, multivariate image analysis, image quantification and image fusion. We are currently developing a Matlab-based Graphical User Interface (GUI), that includes all of these steps (Figure 2). The goals of the GUI include managing images from multiple modalities, performing multiple imaging processing steps such as classification and PCA, segmentation, image registration, image fusion, volume reconstruction; providing tools that support flexibility by incorporating new and existing image analysis routines; 
and providing a simple, yet powerful user interface. The current status and availability of the GUI will be described.

\section{References}

[1]. K. Artyushkova et al., XPS Imaging. IM Publications: UK (2003) 677.

[2]. K. Artyushkova et al., J. Surf. Anal. 9 (2002) 332.

[3]. This work is partially supported by NSF DMR-0354157, NSF CHE-9613880, NSF CORE

IGERT and UNM Keck Nanofluidics Laboratory. The XPS was funded by a grant from the Keck Foundation and NSF CHE-011374.

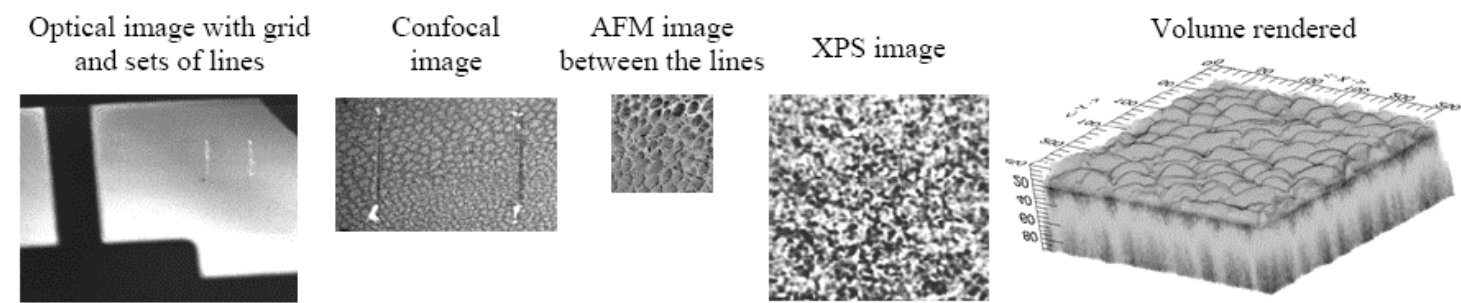

FIG. 1. Marked PS/PMMA sample: Confocal, AFM and XPS images; quantified PS volume

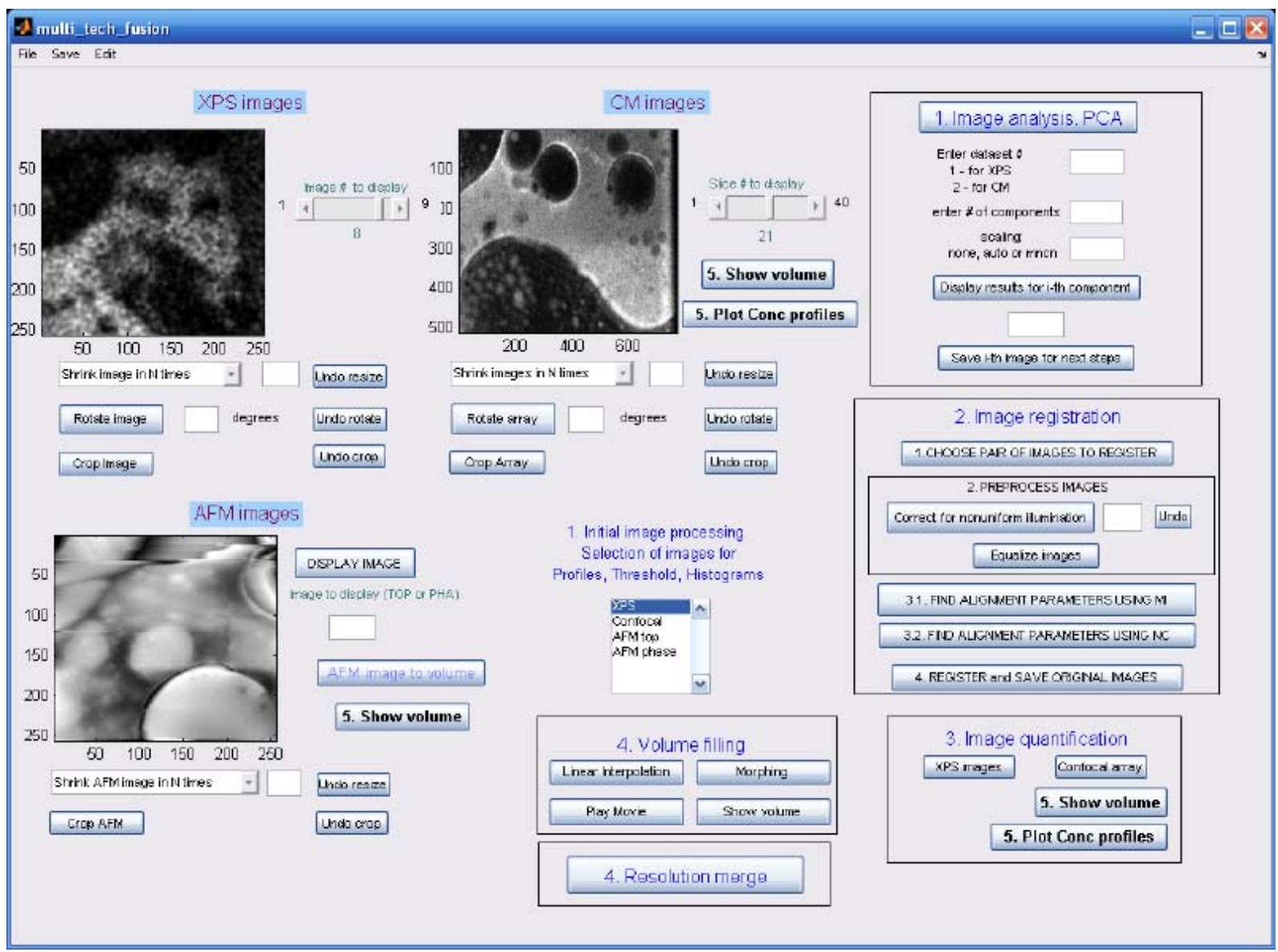

FIG. 2. Example of the Matlab-based Graphical User Interface (GUI) 